\title{
DEGENERATE KERNEL METHOD FOR HAMMERSTEIN EQUATIONS
}

\author{
HIDEAKI KANEKO AND YUESHENG XU
}

\begin{abstract}
The classical method of the degenerate kernel method is applied to numerically solve the Hammerstein equations. Several numerical examples are given to demonstrate the effectiveness of the current method. A brief discussion of a number of methods to decompose the kernel is also included.
\end{abstract}

\section{INTRODUCTION}

This paper is concerned with the problem of finding numerical solutions of the Hammerstein equation

$$
\varphi(x)-\int_{0}^{1} k(x, y) \psi(y, \varphi(y)) d y=f(x), \quad x \in[0,1]
$$

where $k, f$, and $\psi$ are given functions and $\varphi$ is the solution to be determined. Several numerical methods for approximating the solution of (1.1) are known. The classical method of successive approximations was introduced in the 1950's [7]. A variation of Nyström's method was presented in [5]. A new collocationtype method was developed in recent papers [3, 4]. In this paper, we employ the degenerate kernel method, which plays an important role in the study of numerical solutions for the Fredholm integral equations of the second kind. A unified theory to present various degenerate kernel methods within the framework of the projection method was recently presented in [2]. In $\S 2$ we present the degenerate kernel method and consider the problem of existence and uniqueness of the solution of the new Hammerstein equation associated with the degenerate kernel. The problem of convergence of the numerical solution is also considered in $\S 2$. Some examples are considered in $\S 3$. In $\S 4$ we approximate a given kernel by several specific degenerate kernels and obtain convergence rates for the corresponding approximate solutions of equation (1.1). The idea of approximating the kernel by Boolean sums is also included in this section. A comparison of the performance of the Boolean sum approximation scheme with that of the tensor product approximation scheme ( $\S 3$, Example 4$)$ will be given in a future paper.

Received February 13, 1989; revised April 23, 1990.

1980 Mathematics Subject Classification (1985 Revision). Primary 65R20, 45G10.

(C) 1991 American Mathematical Society $0025-5718 / 91 \$ 1.00+\$ .25$ per page 
In order to guarantee the existence of a unique solution to equation (1.1), we assume throughout this paper that the following conditions (i)-(iv) be satisfied:

(i) $k(x, y) \in C([0,1] \times[0,1])$;

(ii) $\psi(y, u)$ is continuous in $y \in[0,1]$ and $u \in(-\infty, \infty)$, and

$$
\left\{\int_{0}^{1}|\psi(y, \varphi(y))|^{2} d y\right\}^{1 / 2} \leq A\|\varphi\|_{2},
$$

where \|\|$_{2}$ denotes the $L_{2}$ norm;

(iii) $\psi(y, u)$ satisfies the Lipschitz condition $|\psi(y, u)-\psi(y, v)| \leq$ $B|u-v|$

(iv) $k$ is bounded by $|k(x, y)|<C$ with $B C<1$.

Under these conditions there exists a unique solution in $L_{2}[0,1]$ for equation (1.1). This can be proved by the Banach contraction mapping principle. To obtain a higher-order convergence rate, we need to assume higher-order smoothness conditions on $k$.

As the first example in $\S 4$ shows, the method of degenerate kernel described in this paper may be applied to integral equations with multiple solutions. Hence, conditions (i)-(iv), which guarantee the global uniqueness of the solution, are perhaps too strong for deciding whether our method is applicable. A reader interested in a more local property of the solution can find a related discussion in [3].

\section{Degenerate KeRnel Method}

Suppose that $k_{n}(x, y)$ is an approximation of the kernel $k(x, y)$, and that it is of a degenerate kernel form,

$$
k_{n}(x, y)=\sum_{i=1}^{n} B_{i}(x) C_{i}(y),
$$

where the set of functions $\left\{B_{i}(x)\right\}$ is assumed to be linearly independent. We also assume that

$$
\left\{\int_{0}^{1} \int_{0}^{1}\left|k(x, y)-k_{n}(x, y)\right|^{2} d x d y\right\}^{1 / 2} \rightarrow 0 \quad \text { as } n \rightarrow \infty .
$$

It is natural to expect the solutions of the following equation (2.3) associated with the degenerate kernels $k_{n}(x, y)$ to converge to the exact solution of equation (1.1),

$$
\varphi_{n}(x)-\int_{0}^{1} k_{n}(x, y) \psi\left(y, \varphi_{n}(y)\right) d y=f(x), \quad x \in[0,1] .
$$

To solve equation (2.3), substitute $k_{n}(x, y)$ into (2.3) and find

$$
\varphi_{n}(x)-\sum_{i=1}^{n} B_{i}(x) \int_{0}^{1} C_{i}(y) \psi\left(y, \varphi_{n}(y)\right) d y=f(x), \quad x \in[0,1] .
$$


Let

$$
\alpha_{i}=\int_{0}^{1} C_{i}(y) \psi\left(y, \varphi_{n}(y)\right) d y .
$$

Then $\varphi_{n}$ can be expressed as

$$
\varphi_{n}(x)=f(x)+\sum_{i=1}^{n} \alpha_{i} B_{i}(x)
$$

where the $\alpha_{i}$ 's are constants to be determined. Once the $\alpha_{i}$ 's have been obtained, (2.6) gives the solution of (2.3). Upon substitution of (2.6) into (2.5), we have

$$
\alpha_{j}=\int_{0}^{1} C_{j}(y) \psi\left(y, f(y)+\sum_{i=1}^{n} \alpha_{i} B_{i}(y)\right) d y \quad \text { for } j=1,2, \ldots, n .
$$

Define

$$
F_{j}\left(\alpha_{1}, \alpha_{2}, \ldots, \alpha_{n}\right)=\int_{0}^{1} C_{j}(y) \psi\left(y, f(y)+\sum_{i=1}^{n} \alpha_{i} B_{i}(y)\right) d y .
$$

Then (2.7) becomes a system of nonlinear algebraic equations

$$
\left[\begin{array}{c}
\alpha_{1} \\
\alpha_{2} \\
\vdots \\
\alpha_{n}
\end{array}\right]=\left[\begin{array}{c}
F_{1}\left(\alpha_{1}, \alpha_{2}, \ldots, \alpha_{n}\right) \\
F_{2}\left(\alpha_{1}, \alpha_{2}, \ldots, \alpha_{n}\right) \\
\vdots \\
F_{n}\left(\alpha_{1}, \alpha_{2}, \ldots, \alpha_{n}\right)
\end{array}\right],
$$

or, in vector notation,

$$
\boldsymbol{\alpha}=\mathbf{F}(\boldsymbol{\alpha}),
$$

where $\boldsymbol{\alpha}^{T}=\left(\alpha_{1}, \alpha_{2}, \ldots, \alpha_{n}\right)$ and $\mathbf{F}(\boldsymbol{\alpha})^{T}=\left(F_{1}(\boldsymbol{\alpha}), F_{2}(\boldsymbol{\alpha}), \ldots, F_{n}(\boldsymbol{\alpha})\right)$.

We shall show that the unique solution of equation $(2.10)$ corresponds to the unique solution of equation (2.3) for each $n$ under some mild assumptions. Equation (2.10) can be solved by a number of standard numerical methods, such as Newton's iterative method or modified Newton's iterative method.

Now we consider the existence of a unique solution of (2.3). We recall that assumptions (i)-(iv) are standing assumptions throughout the rest of this paper.

Theorem 1. Let $k_{n}(x, y) \in C([0,1] \times[0,1])$ satisfy condition (2.2). Then there exists an integer $N>0$ such that, for each $n>N$, equation (2.3) has a unique solution $\varphi_{n} \in L_{2}[0,1]$.

Proof. In view of assumption (iv) and condition (2.2), there exists an integer $N$ such that for each $n>N$,

$$
\left\{\int_{0}^{1} \int_{0}^{1}\left|k_{n}(x, y)\right|^{2} d x d y\right\}^{1 / 2} \leq C .
$$


For each $n>N$, define

$$
\left(T_{n} \varphi\right)(x)=\int_{0}^{1} k_{n}(x, y) \psi(y, \varphi(y)) d y
$$

and

$$
\left(\widehat{T}_{n} \varphi\right)(x)=\left(T_{n} \varphi\right)(x)+f(x) .
$$

It is straightforward to verify from (ii) that $\left\|T_{n} \varphi\right\|_{2} \leq C A\|\varphi\|_{2}$ for all $\varphi \in$ $L_{2}[0,1]$. Hence, $T_{n}$ is a bounded nonlinear operator. Also, for $\varphi_{1}, \varphi_{2} \in$ $L_{2}[0,1]$,

$$
\begin{aligned}
& \left\|\widehat{T}_{n} \varphi_{1}-\widehat{T}_{n} \varphi_{2}\right\|_{2}=\left\|T_{n} \varphi_{1}-T_{n} \varphi_{2}\right\|_{2} \\
& \quad \leq\left\{\int_{0}^{1} \int_{0}^{1}\left|k_{n}(x, y)\right|^{2} d x d y \int_{0}^{1}\left|\psi\left(y, \varphi_{1}(y)\right)-\psi\left(y, \varphi_{2}(y)\right)\right|^{2} d y\right\}^{1 / 2} \\
& \quad \leq C B\left\|\varphi_{1}-\varphi_{2}\right\|_{2} \quad \text { by (iii) and (iv). }
\end{aligned}
$$

Since $0<C B<1$ by assumption, $\widehat{T}_{n}$ is a contraction operator. It follows that there is a unique fixed point of $\widehat{T}_{n}$, which is of course the unique solution of equation (2.3), and $\varphi_{n} \in L_{2}[0,1]$.

Theorem 2. Let

$$
M=B\left\{\sum_{i=1}^{n} \int_{0}^{1}\left|B_{i}(x)\right|^{2} d x\right\}^{1 / 2}\left\{\sum_{i=1}^{n} \int_{0}^{1}\left|C_{i}(x)\right|^{2} d x\right\}^{1 / 2}
$$

and assume $M<1$. Then the nonlinear algebraic equations (2.10) have a unique solution $\boldsymbol{\alpha}^{*}=\left(\alpha_{1}^{*}, \alpha_{2}^{*}, \ldots, \alpha_{n}^{*}\right)$, and

$$
\varphi_{n}(x)=f(x)+\sum_{i=1}^{n} \alpha_{i}^{*} B_{i}(x)
$$

is the unique solution of equation (2.3).

Proof. Define, as usual, the discrete $l_{2}$ norm by $\|\boldsymbol{\alpha}\|_{l_{2}}=\left\{\sum_{i=1}^{n}\left|\alpha_{i}\right|^{2}\right\}^{1 / 2}$ for $\boldsymbol{\alpha}=\left(\alpha_{1}, \alpha_{2}, \ldots, \alpha_{n}\right)^{T} \in l_{2}(n)$. For $\boldsymbol{\alpha}^{(1)}=\left(\alpha_{1}^{(1)}, \ldots, \alpha_{n}^{(1)}\right)$ and $\boldsymbol{\alpha}^{(2)}=$ $\left(\alpha_{1}^{(2)}, \ldots, \alpha_{n}^{(2)}\right)$,

$$
\begin{aligned}
\left\|\mathbf{F}\left(\boldsymbol{\alpha}^{(1)}\right)-\mathbf{F}\left(\boldsymbol{\alpha}^{(2)}\right)\right\|_{l_{2} \leq} \leq & \left\{\sum_{i=1}^{n} \int_{0}^{1}\left|B_{i}(x)\right|^{2} d x\right\}^{1 / 2} \\
& \times\left\{\sum_{i=1}^{n} \int_{0}^{1}\left|C_{i}(x)\right|^{2} d x\right\}^{1 / 2}\left\|\boldsymbol{\alpha}^{(1)}-\boldsymbol{\alpha}^{(2)}\right\|_{l_{2}} .
\end{aligned}
$$

Consequently, $\mathbf{F}$ is a contraction operator in $l_{2}(n)$, since $M<1$. Hence, $\mathbf{F}$ has a unique fixed point $\boldsymbol{\alpha}^{*}$, i.e., $\mathbf{F}\left(\boldsymbol{\alpha}^{*}\right)=\boldsymbol{\alpha}^{*}$. For this $\boldsymbol{\alpha}^{*}$, it is obvious that $\varphi_{n}(x)$ defined by $(2.12)$ is a solution of $(2.3)$, and by Theorem $1, \varphi_{n}(x)$ is the unique solution of (2.3). 
Theorem 3. For

$$
\left\|K-K_{n}\right\|_{2} \equiv\left\{\int_{0}^{1} \int_{0}^{1}\left|k(x, y)-k_{n}(x, y)\right|^{2} d x d y\right\}^{1 / 2}
$$

assume that condition (2.2) holds. Then,

$$
\begin{gathered}
\left\|\varphi-\varphi_{n}\right\|_{2} \leq \frac{A\|\varphi\|_{2}}{1-C B}\left\|K-K_{n}\right\|_{2}, \\
\left\|\varphi-\varphi_{n}\right\|_{2} \leq \frac{A\left\|\varphi_{n}\right\|_{2}}{1-C B}\left\|K-K_{n}\right\|_{2} .
\end{gathered}
$$

Therefore, $\varphi_{n}$ converges to $\varphi$ in $L_{2}[0,1]$.

Proof. Since

$$
\begin{aligned}
\varphi(x)-\varphi_{n}(x)= & \int_{0}^{1}\left[k(x, y)-k_{n}(x, y)\right] \psi(y, \varphi(y)) d y \\
& +\int_{0}^{1} k_{n}(x, y)\left[\psi(y, \varphi(y))-\psi\left(y, \varphi_{n}(y)\right)\right] d y,
\end{aligned}
$$

we have

$$
\left\|\varphi-\varphi_{n}\right\|_{2} \leq A\left\|K-K_{n}\right\|_{2}\|\varphi\|_{2}+C B\left\|\varphi-\varphi_{n}\right\|_{2} .
$$

By virtue of the condition $0<C B<1$,

$$
\left\|\varphi-\varphi_{n}\right\|_{2} \leq \frac{A\|\varphi\|_{2}}{1-C B}\left\|K-K_{n}\right\|_{2} .
$$

The proof of (2.15) is quite similar. Since $\left\|K-K_{n}\right\|_{2} \rightarrow 0$ as $n \rightarrow \infty$, $\left\|\varphi-\varphi_{n}\right\|_{2} \rightarrow 0$ as $n \rightarrow \infty$.

The inequalities (2.14) and (2.15) can be viewed as a priori and a posteriori estimates, respectively, of the error of the approximate solution.

\section{EXAMples}

Example 1. First we substantiate the claim made earlier that uniqueness of the solution is not necessary for the current method to be applicable. Consider [5]

$$
\varphi(x)-\int_{0}^{1} x y \varphi^{2}(y) d y=\frac{3}{4} x, \quad x \in[0,1] .
$$

The actual solutions are $\varphi_{1}(x)=x$ and $\varphi_{2}(x)=3 x$. The kernel function is already degenerate and we obviously take $B_{1}(x)=x$ and $C_{1}(y)=y$. Upon substituting $\varphi_{n}(x)=\frac{3}{4} x+\alpha x$ into (2.5), we obtain $\alpha^{2}-\frac{5}{2} \alpha+\frac{9}{16}=0$. Solving this equation, the exact solutions are obtained.

Example 2. Consider

$$
\varphi(x)-\int_{0}^{1}(1+x y) \varphi^{2}(y) d y=\frac{3}{4} x-\frac{1}{3}, \quad x \in[0,1] .
$$

Again, the kernel is degenerate. We take $B_{1}(x)=1$ and $B_{2}(x)=x$. The $C_{i}$ 's are chosen in the same way. Upon substituting $\varphi_{n}=\left(\frac{3}{4} x-\frac{1}{3}\right)+\alpha_{1}+\alpha_{2} x$ into 
(2.5) and solving the resulting equations, we obtain $\alpha_{1}=\frac{1}{3}$ and $\alpha_{2}=\frac{1}{4}$. The exact solution $\varphi(x)=x$ is obtained by substituting these values of $\alpha_{1}$ and $\alpha_{2}$ in the expression $\varphi_{n}$ above.

Example 3. Consider [5]

$$
\varphi(x)-\int_{0}^{1} e^{x y} e^{-\varphi^{2}(y)} d y=\sqrt{x}-\frac{e^{x-1}-1}{x-1}, \quad x \in[0,1] .
$$

The exact solution is $\varphi(x)=\sqrt{x}$. We approximate $e^{x y}$ by $1+x y+(x y)^{2} / 2+$ $\cdots+(x y)^{n} / n !$. An approximate solution

$$
\varphi_{n}(x)=\sqrt{x}-\frac{e^{x-1}-1}{x-1}+\alpha_{1}+\alpha_{2} x+\alpha_{3} \frac{x^{2}}{\sqrt{2}}+\cdots+\alpha_{n+1} \frac{x^{n}}{\sqrt{n !}}
$$

is obtained by solving (2.5). For example, for $n=2$, we obtain $\alpha_{1}=.634038$, $\alpha_{2}=.266290, \alpha_{3}=.114898$. The errors $e_{n}$ for different values of $n$ are listed below. The $e_{n}$ 's are approximated by the composite Simpson's formula.

$$
\begin{array}{cc}
n & e_{n}=\left\|\varphi-\varphi_{n}\right\|_{2} \\
\hline & 7.218 \mathrm{E}-2 \\
3 & 9.847 \mathrm{E}-3 \\
4 & 2.013 \mathrm{E}-3
\end{array}
$$

Example 4. Consider the same equation as in Example 3. Now the kernel is approximated by $\sum_{i=0}^{2^{n}} \sum_{j=0}^{2^{n}} e^{x_{i} y_{j}} B_{i}(x) C_{j}(y)$, where the $B_{i}$ 's and $C_{j}$ 's are linear $B$-splines with knots $0=x_{0}<x_{1}<\cdots<x_{2^{n}}=1$, where $x_{i}=i / 2^{n}$ for $i=0,1, \ldots, 2^{n}$. The approximation

$$
\varphi_{n}(x)=\sqrt{x}-\frac{e^{x-1}-1}{x-1}+\sum_{i=0}^{2^{n}} \alpha_{i} B_{i}(x)
$$

is obtained upon solving (2.5) and gives rise to errors $e_{n}$ shown below.

$$
\begin{array}{cc}
n & e_{n}=\left\|\varphi-\varphi_{n}\right\|_{2} \\
\hline & \\
2 & 6.203 \mathrm{E}-2 \\
3 & 1.553 \mathrm{E}-2 \\
4 & 3.768 \mathrm{E}-3 \\
5 & 9.432 \mathrm{E}-4
\end{array}
$$

\section{APPROXIMATIONS OF THE KERNEL}

The examples in $\S 3$ are mainly concerned with the method of approximating the kernel $k(x, y)$ by degenerate kernels having the form of tensor products of 
univariate functions. In this section, we present another approach, a variation of tensor products, to decompose $k(x, y)$.

Let $L_{x}$ be a projection of $C([0,1] \times[0,1])$ onto $X_{n} \times C[0,1]$, where $X_{n}$ is an $n$-dimensional subspace of $C[0,1]$. Similarly, let $M_{y}$ be a projection of $C([0,1] \times[0,1])$ onto $C[0,1] \times Y_{m}$, where $Y_{m}$ is an $m$-dimensional subspace of $C[0,1]$. Define

$$
R_{x} k(x, y)=k(x, y)-L_{x} k(x, y)
$$

and

$$
R_{y} k(x, y)=k(x, y)-M_{y} k(x, y) .
$$

Then $L_{x} k$ and $M_{y} k$ provide two degenerate kernels that approximate partially $k(x, y)$ with respect to $x$ and $y$, with respective errors $R_{x} k$ and $R_{y} k$. Then $L_{x} M_{y} k$ gives a complete approximation of $k(x, y)$ in both $x$ and $y$, with error term

$$
R\left(L_{x} M_{y}\right) k=R_{x} k+R_{y} k-R_{x} R_{y} k .
$$

Thus, the rate of convergence depends on the approximation powers of $L_{x}$ and $M_{y}$. In order to enhance the speed of convergence, we define the Boolean sum of $L_{x}$ and $M_{y}$ by

$$
L_{x} \otimes M_{y}=L_{x}+M_{y}-L_{x} M_{y} .
$$

Then $\left(L_{x} \otimes M_{y}\right) k$ approximates $k$ with the error

$$
R\left(L_{x} \otimes M_{y}\right) k=R_{x} R_{y} k .
$$

To see (4.5), we have by the definition of $L_{x} \otimes M_{y}$,

$$
\begin{aligned}
k-\left(L_{x} \otimes M_{y}\right) k & =\left(I-L_{x}\right) k+\left(I-M_{y}\right) k-\left(I-L_{x} M_{y}\right) k \\
& =R_{x} k+R_{y} k-\left(R_{x} k+R_{y} k-R_{x} R_{y} k\right)=R_{x} R_{y} k .
\end{aligned}
$$

Thus, this new approximation of $k$ by $\left(L_{x} \otimes M_{y}\right) k$ enhances the rate of convergence. More specifically, let $X_{n}$ be the space of spline functions of degree $n_{1}-1$ with knots at $0=x_{0}<x_{1}<\cdots<x_{k_{1}}<x_{k_{1}+1}=1$, where $n_{1}+k_{1}=: n$. Similarly, let $Y_{m}$ be the space of spline functions of degree $m_{1}-1$ with knots at $0=y_{0}<y_{1}<\cdots y_{k_{2}}<y_{k_{2}+1}=1$, where $m_{1}+k_{2}=: m$. Chapters 4 and 12 of [6] are particularly useful in relation to our current discussion.

Let $L_{x}$ and $M_{y}$ be the interpolatory operators onto $X_{n}$ and $Y_{m}$, respectively. Assuming $k \in C^{n_{1}}[0,1] \times C[0,1]$, we have

$$
\sup \left|R_{x} k(x, y)\right| \leq\left|C_{1}(y)\right| h_{x}^{n_{1}} \leq C_{1} h_{x}^{n_{1}}, \quad C_{1} \text { a constant }
$$

and similarly, when $k \in C[0,1] \times C^{m_{1}}[0,1]$, then

$$
\sup \left|R_{y} k(x, y)\right| \leq\left|C_{2}(x)\right| h_{y}^{m_{1}} \leq C_{2} h_{y}^{m_{1}}, \quad C_{2} \text { a constant, }
$$

where $h_{x}=\max \left\{x_{i}-x_{i-1}: i=1,2, \ldots, k_{1}+1\right\}$ and $h_{y}=\max \left\{y_{i}-y_{i-1}: i=\right.$ $\left.1,2, \ldots, k_{2}+1\right\}$. sion.

The following theorem is an immediate consequence of the preceding discus- 
Theorem 4. (i) Let $k \in C^{n_{1}}[0,1] \times C[0,1]$, and let $\varphi_{n}$ be the solution of $(2.3)$ with $k_{n}(x, y):=L_{x} k(x, y)$. Then $\left\|\varphi-\varphi_{n}\right\|_{2} \leq \widetilde{C}_{1} h_{x}^{n_{1}}$.

(ii) Let $k \in C[0,1] \times C^{m_{1}}[0,1]$, and let $\varphi_{m}$ be the solution of (2.3) with $k_{m}(x, y):=M_{y} k(x, y)$. Then $\left\|\varphi-\varphi_{m}\right\|_{2} \leq \widetilde{C}_{2} h_{y}^{m_{1}}$.

(iii) Let $k \in C^{n_{1}}[0,1] \times C^{m_{1}}[0,1]$, and let $\varphi_{n}$ be the solution of $(2.3)$ with $k_{n}(x, y):=L_{x} M_{y} k(x, y)$. Then $\left\|\varphi-\varphi_{n}\right\|_{2} \leq \widetilde{C}_{3} h^{n_{2}}$, where $h=\max \left\{h_{x}, h_{y}\right\}$ and $n_{2}=\min \left\{n_{1}, m_{1}\right\}$.

(iv) Let $k \in C^{n_{1}}[0,1] \times C^{m_{1}}[0,1]$, and let $\varphi_{n}$ be the solution of $(2.3)$ with $k_{n}(x, y)=\left(L_{x} \otimes M_{y}\right) k(x, y)$. Then $\left\|\varphi-\varphi_{n}\right\|_{2} \leq \widetilde{C}_{4} h^{n_{1}+m_{1}}$.

Proof. Proofs of (i)-(iv) of this theorem are quite obvious from the discussion in this section and Theorem 3. We demonstrate the proof for (iv) only. By (4.5), (4.6), and (4.7),

$$
\left\|k-\left(L_{x} \otimes M_{y}\right) k\right\| \leq C_{4} h_{x}^{n_{1}} h_{y}^{m_{1}} .
$$

By Theorem 3,

$$
\left\|\varphi-\varphi_{n}\right\|_{2} \leq \frac{A\left\|\varphi_{n}\right\|_{2}}{1-C B} C_{4} h_{x}^{n_{1}} h_{y}^{m_{1}} \leq \widetilde{C}_{4} h^{n_{1}+m_{1}}, \quad \text { where } \widetilde{C}_{4}=\frac{A C_{4}\left\|\varphi_{n}\right\|_{2}}{1-C B} .
$$

There exists only a small number of papers which use the Boolean sum approximation technique. These papers are mainly in the area of approximation of eigenvalues of certain operators (see, e.g., [1] and references cited therein).

\section{BIBLIOGRAPHY}

1. L. Bamberger and G. Hämmerlin, Spline-blended substitution kernels of optimal convergence, Treatment of Integral Equations by Numerical Methods (C. T. H. Baker and G. F. Miller, eds.), Academic Press, 1982, pp. 47-58.

2. H. Kaneko, A projection method for solving Fredholm integral equations of the second kind, IMACS Appl. Numer. Math. 5 (1989), 333-344.

3. S. Kumar and I. H. Sloan, A new collocation-type method for Hammerstein integral equations, Math. Comp. 48 (1987), 585-593.

4. S. Kumar, A discrete collocation-type method for Hammerstein equations, SIAM J. Numer. Anal. 25 (1988), 328-341.

5. L. J. Lardy, A variation of Nyström's method for Hammerstein equations, J. Integral Equations 3 (1981), 43-60.

6. L. L. Schumaker, Spline functions: Basic theory, Wiley, New York, 1981.

7. F. G. Tricomi, Integral equations, Dover, New York, 1985.

Department of Mathematics and Statistics, Old Dominion University, Norfolk, VirGINIA 23529-0077

Department of Mathematical Sciences, North Dakota State University, Fargo, NORTH DAKOTA 58105 